\title{
Lugano Classification Advanced Stage Childhood Hodgkin Lymphoma AJCC v8
}

National Cancer Institute

\section{Source}

National Cancer Institute. Lugano Classification Advanced Stage Childhood Hodgkin

Lymphoma A/CC v8. NCI Thesaurus. Code C141185.

Advanced stage childhood Hodgkin lymphoma based on the Lugano classification

criteria. It includes stages III and IV. (from AJCC 8th Ed.) 